ISSN 0103-8478

\title{
A sedação sobre os potenciais evocados auditivos em cães
}

\author{
The sedation on the auditory brainstem responses in dogs
}

\author{
Mariana Isa Poci Palumbo ${ }^{\mathrm{I}}$ Mariana Cristina Ramos ${ }^{\mathrm{I}}$ Nadia Crosignani Outeda ${ }^{\mathrm{I}}$ \\ Luiz Antonio de Lima Resende ${ }^{\mathrm{II}}$ José Carlos de Figueiredo Pantoja ${ }^{\mathrm{I}}$ Alexandre Secorun Borges ${ }^{\mathrm{I}^{*}}$
}

RESUMO

O potencial evocado auditivo de tronco encefálico (Brainstem Auditory Evoked Potential - BAEP) avalia a atividade elétrica do sistema auditivo, desde a cóclea até o tronco encefálico, permitindo diagnóstico de surdez em cães. Como alguns animais não permitem a obtenção do BAEP sem contenção química, o objetivo deste trabalho foi analisar a influência da sedação com morfina e acepromazinano BAEP de 16 cães com audição normal. Os potenciais foram obtidos antes e durante a sedação com administração intramuscular de morfina $\left(0,5 \mathrm{mgkg}^{-1}\right)$ e acepromazina $\left(0,05 \mathrm{mgkg}^{-1}\right)$. O protocolo de sedação utilizado permitiu contenção efetiva e segurança dos animais. A sedação causou prolongamento nas latências das ondas II, III e intervalos I-III e I-V, mas não dificultou as suas identificações. O uso deste protocolo de sedação não interferiu com a interpretação do potencial evocado e pode ser útil em cães. Pelo que se pode observar na literatura compilada, este é o primeiro estudo avaliando a influência da sedação no BAEP em cães no Brasil.

Palavras-chave: teste eletrofisiológico, anestesia, morfina, acepromazina, audição, medicina veterinária.

\section{ABSTRACT}

Brainstem auditory evoked potential (BAEP) reflects the electrical activity along the auditory pathway, from the cochlea to the brainstem, and contributes for the diagnosis of deafness in dogs. BAEP recording may require chemical restraint in some cases, so this study was designed to analyze the impact of sedation with morphine and acepromazine on the BAEP recordings of 16 dogs with normal hearing. BAEPs were recorded before and during sedation with a combination of morphine $\left(0.5 \mathrm{mg} \mathrm{kg}^{-1}\right)$ and acepromazine $\left(0.05 \mathrm{mgkg}^{-1}\right)$ given intramuscularly. The protocol employed allowed safe and effective animal restraint. Sedation increased the latency of waves II and III and intervals I-III and $I-V$ but did not interfere with wave identification. and showed to be safe in the dogs tested. Based on the current literature this is the first study which assessed the impact of sedation on BAEPs in dogs in Brazil.

Key words: electrophysiological test, anesthesia, morphine, acepromazine, hearing, veterinary medicine.

\section{INTRODUÇÃO}

A importância da audição no bem-estar e interação social dos cães (COX, 2002) e o notável aumento da preocupação das pessoas com o estado de saúde de seus animais de companhia (COE et al., 2007) estimularam a utilização de técnicas precisas e não invasivas para o diagnóstico de surdez. Um exemplo é o potencial evocado auditivo de tronco encefálico (Brainstem Auditory Evoked Potential BAEP) (WEBB, 2009).

A audição refere-se à capacidade de percepção dos sons pelos animais. Os sons são vibrações do ambiente que estimulam um conjunto de receptores e geram potenciais de ação que são transmitidos por vias ascendentes até o córtex encefálico, onde a informação é processada e percebida conscientemente (KAY et al., 1984;LENT, 2002).

Não é possível diagnosticar a perda de audição apenas com a inspeção direta de um animal (COX, 2002). No exame clínico, a porção auditiva do oitavo nervo craniano pode ser testada, com o animal de olhos vendados, realizando estímulos auditivos. O animal deve virar a cabeça na direção do som. Lesões

'Universidade Estadual Paulista "Júlio de Mesquita Filho" (UNESP), Rubião Júnior s/n, 18618-000, Botucatu, SP, Brasil. E-mail: asborges@fmvz.unesp.br.*Autor para correspondência.

"Departamento de Neurologia, Psicologia e Psiquiatria, Faculdade de Medicina de Botucatu (FMB), Botucatu, SP, Brasil. Recebido 31.10.12 Aprovado 09.10.13 Devolvido pelo autor 11.03.14 CR-2012-1064.R1 
bilaterais resultam em ausência de resposta. Entretanto, este tipo de avaliação pode não ser efetivo em situações de lesões unilaterais (MUNRO \& COX, 1997; COX, 2002; MARCONDES, 2008). Outro problema é a inviabilidade do teste em filhotes, cães agitados, desatentos ou com alteração do nível de consciência (STRAIN, 1999; PONCELET et al., 2002).

Uma avaliação objetiva e precisa do sistema auditivo inclui a utilização de testes eletrodiagnósticos (STRAIN, 2003; WILSON \& MILLS, 2005; MARCONDES, 2008). O BAEP é um teste objetivo e não invasivo que indica a presença ou ausência de função auditiva, sendo considerado o "padrão ouro" nos testes auditivos em animais, detectando a surdez bilateral e unilateral, além de poder ser realizado no animal acordado ou anestesiado (COX, 2002; STRAIN, 2003; WILSON \& MILLS, 2005).

O BAEP é um teste eletrodiagnóstico classificado como potencial evocado. Potenciais evocados são respostas elétricas de um sistema sensorial, registrados após estímulos (CHIAPPA, 1997). O BAEP pode ser gravado a partir de um estímulo sonoro aplicado no ouvido por meio de fones (COX, 2002; WILSON \& MILLS, 2005), ou através da estimulação direta da cóclea por meio da vibração do osso mastoide com um estimulador ósseo (MUNRO et al., 1997; STRAIN 2003).

As atividades elétricas das vias auditivas são captadas com a utilização de três eletrodos subcutâneos: ativo, referência e terra (WEBB, 2009). A resposta elétrica captada pelos eletrodos é enviada para o pré-amplificador, a diferença de voltagem entre os eletrodos do vértice da cabeça e da orelha testada é amplificada e, em seguida, digitalizada (WILSON \& MILLS, 2005).

No resultado final desse processo, é obtido um traçado que consiste de quatro a sete ondas. Cada onda está associada a uma estrutura particular do sistema auditivo (WEBB, 2009). Estudos em cães comparando os resultados do teste com outros achados de diagnóstico mostraram que as ondas I a III são geralmente formadas pela atividade do nervo vestibulococlear, núcleo coclear e complexo olivar superior, respectivamente. Enquanto as ondas IV a VII são formadas pela atividade de uma ou mais das seguintes estruturas: núcleo do lemnisco lateral, colículo inferior ou núcleo geniculado medial (WILSON \& MILLS, 2005). Inferências semelhantes foram obtidas em humanos (CHIAPPA, 1997).

O BAEP pode ser utilizado para avaliar a atividade dos neurônios relacionados à audição, mas não pode ser utilizado para comprovar o conceito comportamental de audição, pois seu perfeito funcionamento necessita da integridade de outras estruturas, além das avaliadas nesse teste (WILSON \& MILLS, 2005).

$\mathrm{O}$ teste auditivo pode ser realizado com o animal acordado (STRAIN, 1999; WEBB, 2009), porém, a sedação pode ser necessária em alguns pacientes, pois os registros podem apresentar interferência pelo excessivo movimento muscular (MYERS et al., 1985), principalmente pelos músculos do pescoço e mandíbula. Isso se deve ao fato de a atividade muscular ser mensurada em milivolts, enquanto a atividade neuronal captada pelo BAEP é mensurada em microvolts (WILSON \& MILLS, 2005).

Alguns anestésicos podem alterar a latência e amplitude das ondas, enquanto outros não (WILSON \& MILLS, 2005). MARSHALL (1985), relatou a utilização de acepromazina na dose de $0,55 \mathrm{mgkg}^{-1}$ sem alterações nos traçados. MYERS et al. (1985) utilizaram metoxiflurano inalatório a 3\% e relataram o prolongamento da latência das ondas. Outros autores (MUNRO \& COX, 1997; MUNRO et al., 1997; PONCELET et al., 2002; POMIANOWSKI \& ADAMIAK, 2010) utilizaram diferentes protocolos de sedação sem avaliar se houve alguma interferência nos resultados. Tendo em vista que alguns animais não permitem a realização do BAEP sem contenção química, o objetivo deste trabalho foi analisar a influência da sedação com morfina e acepromazina nos potenciais evocados auditivos de tronco encefálico de cães com audição normal.

\section{MATERIAL E MÉTODOS}

Foram utilizados 16 cães adultos (10 machos e 6 fêmeas) de diferentes raças (13 sem raça definida, 2 labradores e 1 beagle), oriundos de proprietários que forneceram consentimento prévio para participação no experimento. Para evitar alterações decorrentes da idade, apenas animais com idade entre 1 e 5 anos foram incluídos no estudo. Cada cão foi clinicamente avaliado, incluindo realização de exame neurológico, segundo MARCONDES (2008), e exame otoscópico, segundo COLE (2004). Os donos foram questionados sobre histórico de uso de agentes ototóxicos e avaliação subjetiva da audição. Animais com alterações neurológicas, presença de ácaros ou cerúmen em excesso no conduto auditivo externo, ou com qualquer outra anormalidade foram excluídos do estudo. Para avaliar a influência da sedação nos potenciais evocados auditivos, o teste foi realizado antes e 15 minutos após a administração intramuscular de morfina $\left(0,5 \mathrm{mgkg}^{-1}\right)$ e acepromazina 
$\left(0,05 \mathrm{mgkg}^{-1}\right)$. Todos os animais estavam em jejum alimentar de 8 horas e hídrico de duas horas. Foram avaliadas as frequências cardíaca e respiratória, além da temperatura retal, antes e durante o procedimento. Os animais foram monitorados até a completa recuperação.

Potenciais evocados foram obtidos no Laboratório de Eletroneurodiagnóstico do Departamento de Clínica Veterinária, FMVZ- UnespBotucatu. Os animais foram mantidos em posição quadrupedal ou decúbito esternal. Foram utilizados eletrodos monopolares de inserção (spes medica ${ }^{\mathbb{R}}$ ) e não houve necessidade da realização de tricotomia ou uso de pasta condutora. Os eletrodos de registro foram colocados na região rostral ao tragus da orelha a ser testada (G1) e o de referência (G2) foi posicionado no vértice do crânio (na posição $\mathrm{Cz}$ da eletroencefalografia) (KAYet al., 1984). O eletrodo terra foi colocado na região cervical dorsal (EGER \& LINDSAY, 1997) (Figuras 1 e 2). Foram utilizados filtros com banda passante de $200 \mathrm{~Hz}$ a $3.000 \mathrm{~Hz}$, sensibilidade de $2,5 \mu \mathrm{Vcm}^{-1}$ e varredura de $1 \mathrm{mscm}^{-1}$. Fones de ouvido externos foram posicionados sobre as orelhas dos animais. Utilizaram-se "cliques" de rarefação de $0,2 \mathrm{~ms}$ de duração, intensidade de $85 \mathrm{~dB}$, apresentados na frequência de $13 \mathrm{~Hz}$, com mascaramento de ruído branco contralateral de $40 \mathrm{~dB}$. Em cada lado, foram obtidas pelo menos duas séries de 500 promediações, utilizando-se equipamento ViasysHealthcare ${ }^{\circledR}$, modelo Teca Synergy de dois canais.

Foram avaliadas as latências em milissegundos das ondas I, II, III e V, bem como dos intervalos I-III, III-V e I-V para cada orelha dos cães. A distribuição das variáveis foi analisada para escolha dos métodos analíticos, as análises gráficas (histogramas e gráficos quantil-quantil) evidenciaram desvios significantes de um padrão Gaussiano de distribuição. Dessa forma, método não paramétrico para medidas repetidas (amostras pareadas) foi adotado para a análise. Foi utilizado o Teste de Postos Sinalizados de Wilcoxon (PROC NPAR1WAY; SAS Institute, 2009) para comparação dos resultados entre os lados esquerdo e direito, e antes e durante a sedação.

\section{RESULTADOS E DISCUSSÃO}

Nenhum animal teve complicações e alterações significativas nos parâmetros frequência cardíaca, respiratória e temperatura. Foi possível obtenção de registros de boa qualidade nos 16 animais, em ambas as orelhas, antes e durante a sedação. A figura 3 exemplifica o potencial evocado auditivo obtido antes e durante a sedação em um cão sem raça definida. Não houve diferença estatística significante entre os resultados do lado esquerdo e direito ( $\mathrm{P}>0,36$ para todas as variáveis estudadas) e os resultados foram agrupados, totalizando 32 exames. Em todos os exames, foi possível identificação das ondas I, II, III e V. As médias e desvio padrão das latências das ondas antes e durante a sedação estão representados na tabela 1 .

Ao comparar a latência das ondas antes e durante a sedação, observou-se que as latências das ondas II e III, bem como dos intervalos I-III e I-V, foram estatisticamente maiores durante a sedação. Porém, não foram observadas diferenças para as latências das ondas I e V, bem como intervalo III-V.

Não há associação do sexo dos animais com o resultado do BAEP em cães (MARSHALL, 1985) e, por esse motivo, esta variável não foi levada em consideração na identificação das ondas. Sabe-se que a idade pode interferir nos potenciais obtidos. Filhotes de cães e gatos não nascem com o sistema auditivo completamente desenvolvido (MEIJ

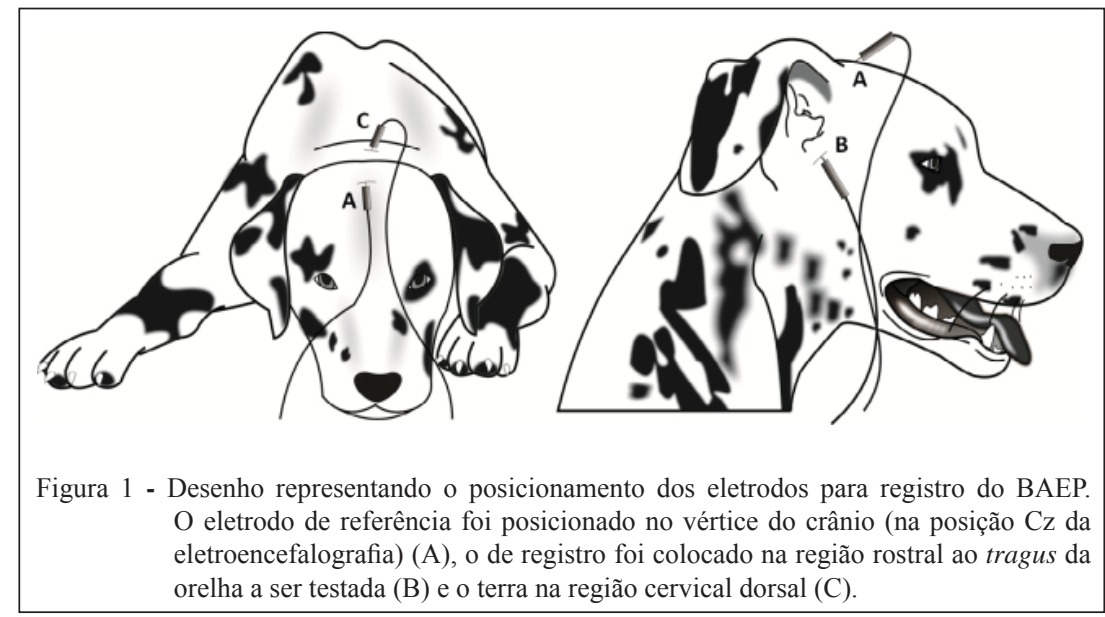

Ciência Rural, v.44, n.5, mai, 2014. 


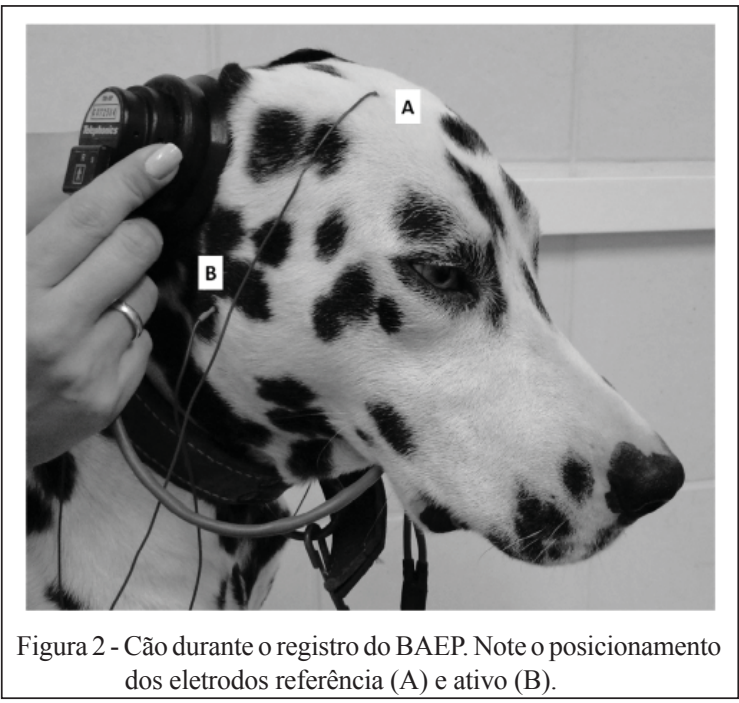

et al., 1992) e os valores de latências e amplitudes semelhantes a adultos são obtidos com seis a oito semanas de idade (PONCELET et al., 2002). Segundo HAAR et al. (2008), a perda da audição relacionada com a idade em cães acontece por volta dos 8 a 10 anos de idade (surdez senil). Para evitar alterações decorrentes da idade, apenas animais com idades entre 1 e 5 anos foram estudados.Em medicina humana, são usados eletrodos de superfície com discos metálicos aderidos à pele, com auxílio de fita adesiva e com pasta condutiva entre o disco e a pele. $\mathrm{O}$ registro dos potenciais requer um contato com baixa impedância entre os eletrodos e a pele do animal. Por ter uma quantidade excessiva de pelos, é difícil conseguir a impedância ideal para registro dos potenciais com uso de eletrodos de superfície em cães (STRAIN, 2011). Por esse motivo, os trabalhos de BAEP em animais utilizam eletrodos de agulha de inserção (MARSHALL, 1985; FAMULA et al., 1996; EGER, LINDSAY, 1997; HAAR et al., 2008). Os cães não demonstraram incômodo com o uso dos eletrodos de inserção no presente estudo.

A maioria dos animais não requer contenção química para a realização do exame, entretanto, o uso de medicação sedativa faz com que o ruído de fundo (da atividade cortical) interfira menos, evitando artefatos e facilitando a identificação das ondas (LASMAR et al., 1994). Observou-se no presente trabalho que a obtenção do BAEP com os animais sedados permitiu a obtenção de traçados com menor interferência. As variações de latências e intervalos entre os grupos acima descritos foram mínimas. As diferenças estatísticas observadas podem ser explicadas pelo fato de os desvios padrão serem muito pequenos, o que aumenta a sensibilidade do teste estatístico para detectar diferenças entre os achados. Também existe a possibilidade de a sedação causar um atraso na velocidade de transmissão dos potenciais de ação, mas seria esperada latência maior de todas as ondas registradas durante a sedação. Acredita-se que essa pequena variação não seja

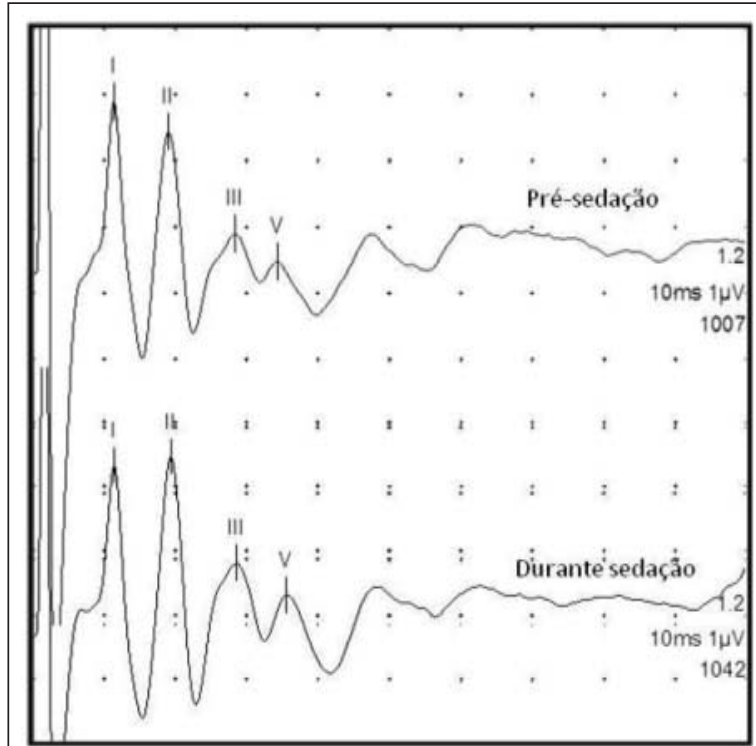

Figura 3 - Potencial evocado auditivo realizado antes e durante a sedação em cão sem raça definida. Note a marcação das ondas I, II, III e V (Divisão vertical: $1 \mu \mathrm{V}$; divisão horizontal: 1 milissegundo).

Ciência Rural, v.44, n.5, mai, 2014. 
Tabela 1 - Média e desvio padrão das latências absolutas das ondas I, II, III e V e dos intervalos I-III, III-V e I-V em milissegundos dos exames realizados antes e durante a sedação. Médias seguidas de letras diferentes na mesma linha indicam diferença estatística entre os grupos $(\mathrm{P} \leq 0,05)$.

\begin{tabular}{|c|c|c|c|c|c|}
\hline \multirow{2}{*}{ Onda } & & çăo- & -------D & dação----- & \multirow{2}{*}{$\mathrm{P}$} \\
\hline & Média (ms) & Desvio Padrão & Média (ms) & Desvio Padrão & \\
\hline I & $1,15^{\mathrm{a}}$ & 0,10 & $1,16^{\mathrm{a}}$ & 0,09 & 0,94 \\
\hline II & $1,92^{\mathrm{a}}$ & 0,10 & $1,96^{\mathrm{b}}$ & 0,10 & $<0,001$ \\
\hline III & $2,67^{\mathrm{a}}$ & 0,28 & $2,70^{\mathrm{b}}$ & 0,18 & 0,01 \\
\hline V & $3,55^{\mathrm{a}}$ & 0,29 & $3,57^{\mathrm{a}}$ & 0,19 & 0,09 \\
\hline I-III & $1,51^{\mathrm{a}}$ & 0,25 & $1,54^{\mathrm{b}}$ & 0,16 & 0,02 \\
\hline III-V & $0,88^{\mathrm{a}}$ & 0,20 & $0,87^{\mathrm{a}}$ & 0,19 & 0,82 \\
\hline $\mathrm{I}-\mathrm{V}$ & $2,37^{\mathrm{a}}$ & 0,31 & $2,39^{b}$ & 0,23 & 0,04 \\
\hline
\end{tabular}

biologicamente relevante e que não interfira na identificação dos animais com surdez, porém, para a interpretação dos resultados, é importante considerar se o animal foi sedado.

Não foram encontrados outros trabalhos com uso de acepromazina e morfina para realização do BAEP em cães. As latências observadas neste estudo foram semelhantes aos resultados descritos por outros autores, que realizaram anestesia com diferentes fármacos para registro do BAEP. KANGet al. (2008) utilizaram medetomidina a $30 \mu \mathrm{gkg}^{-1}$ pela via intramuscular, PONCELET (2002) e BIANCHI et al. (2006) utilizaram este mesmo fármaco, porém em doses diferentes. HAAR et al. (2008) induziram um plano superficial de anestesia com medetomidina (100 $\left.\mu \mathrm{gkg}^{-1} \mathrm{IV}\right)$ seguido de propofol $\left(1 \mathrm{mgkg}^{-1} \mathrm{IV}\right)$. POMA et al. (2008) utilizou sedação com acepromazina, $0,3 \mathrm{mgkg}^{-1}$ e hidromorfina a $0,1 \mathrm{mgkg}^{-1}$ pela via intravenosa e FAMULA et al. (1996) administrou $0,1 \mathrm{mgkg}^{-1}$, via intravenosa. Esses pesquisadores utilizaram a anestesia nos experimentos, porém, não compararam os valores das latências antes e durante a anestesia.

MYERS et al. (1985) realizaram um trabalho avaliando os efeitos da anestesia com metoxifluorano no BAEP e não observaram diferenças significativas entre as latências das ondas registradas das orelhas direita e esquerda, tanto dos animais anestesiados quanto dos não anestesiados, o que foi compatível com o presente estudo. No presente estudo, a sedação com acepromazina e morfina prolongou a latência das ondas II e III, bem como dos intervalos I-III e I-V. Esse resultado foi diferente do observado por MYERS et al. (1985), que evidenciou aumento significativo das latências de todas as ondas registradas nos animais anestesiados com metoxifluorano.Também foi observado que houve diminuição na variabilidade das latências das ondas durante a sedação. Exceto para a onda II, para a qual a mesma variabilidade foi observada antes e durante a sedação, houve uma diminuição entre 5 e $36 \%$ na variabilidade de todas as ondas (Tabela 1), possivelmente, devido à diminuição de interferências que ocorre com o animal acordado.

\section{CONCLUSÃO}

O protocolo de sedação com morfina $(0,5 \mathrm{mg}$ $\left.\mathrm{kg}^{-1}\right)$ e acepromazina $\left(0,05 \mathrm{mg} \mathrm{kg}^{-1}\right)$ se mostrou útil na realização do BAEP por possibilitar uma contenção efetiva e segura dos cães durante o exame. O uso deste protocolo de sedação não interfere na interpretação do potencial evocado e pode ser útil em cães muito agitados. A sedação causou um prolongamento na latência das ondas II, III e intervalos I-III e I-V, sem dificultar a sua identificação.

\section{COMITÊ DE ÉTICA E BIOSSEGURANÇA}

O número do protocolo do comitê de ética é: 117/2009.

\section{AGRADECIMENTOS}

Agradecemos ao apoio fornecido pela Fundação de Amparo à Pesquisa do Estado de São Paulo (FAPESP) (Processos 2009/12290-8 e 2010/00518-1) e ao Conselho Nacional de Desenvolvimento Científico e Tecnológico (Cnpq), pela bolsa de iniciação científica.

\section{REFERÊNCIAS}

BIANCHI, E. et al. N3 potentials in response to high intensity auditory stimuli in animals with suspected cochleo-saccular deafness. Research in Veterinary Science, v.81, p.265269, 2006. Available from: <http://www.ncbi.nlm.nih.gov/ pubmed/16460771>. Accessed:Ago, 01,2012.

CHIAPPA, K.H. Brain stem auditory evoked potentials: methodology. In: CHIAPPA, K.H. (Ed.). Evoked potentials in clinical medicine. Philadelphia: Lippincott-Raven, 1997. p.157-197. 
COE, J.B. et al. A focus group study of veterinarians' and pet owners' perceptions of the monetary aspects of veterinary care. Journal of the American Veterinary Medical Association, v.231, p.1510-1518, 2007. Available from: <http://avmajournals. avma.org/doi/abs/10.2460/javma.231.10.1510?url ver=Z39.88$2003 \&$ rfr_id $=$ ori:rid:crossref.org\&rfr_dat $=$ cr_pub $\% 3$ dpubmed $>$. Accessed: Ago. 01, 2012.doi: 10.2460/javma.231.10.1510.

COLE, L.K. Otoscopic evaluation of the ear canal. Veterinary Clinics of North America - Small Animal Practice, v.34, p.397-410, 2004. Available from: <http://www.ncbi.nlm.nih.gov/ pubmed/15062615>. Accessed: Ago.01, 2012.doi: 10.1016/j. cvsm.2003.10.004

COX, C. Investigation of hearing loss in dogs. In Practice, v.24, p.494-501, 2002. Available from: < http://inpractice.bmj.com/ content/24/9/494.full.pdf + html $>$. Accessed: Ago. 01, 2012.doi: 10.1136/inpract.24.9.494

EGER, C.E.; LINDSAY, P. Effects of otitis on hearing in dogs characterized by brainstem auditory evoked response testing. Journal of Small Animal Practice, v.38, p.380-386, 1997. Available from: $<$ http://www.ncbi.nlm.nih.gov/pubmed/9322176>. Accessed: Ago. 01, 2012.

FAMULA, T.R. et al. A threshold model analysis of deafness in Dalmatians. Mammalian genome, v.7, p.650-653, 1996. Available from: <http://www.springerlink.com/content $/ 2 \mathrm{cx} 18 \mathrm{a} 5 \mathrm{k}$ 1v60nr01/?MUD=MP>. Accessed: Ago. 01, 2012.doi: 10.1007/ s003359900199.

HAAR, G.T. et al. Effects of aging on brainstem responses to toneburst auditory stimuli: a cross-sectional and longitudinal study in dogs. Journal of Veterinary Internal Medicine, v.22, p.937-945, 2008. Available from: < http://onlinelibrary.wiley.com/ doi/10.1111/j.1939-1676.2008.0126.x/abstract;jsessionid=4FFE D56C5CEA103AA645BEB31BC200C2.d03t02?systemMessag $\mathrm{e}=$ Wiley+Online + Library + will + be + disrupted + on $+4+$ August + fro $\mathrm{m}+10 \% 3 \mathrm{~A} 00-12 \% 3 \mathrm{~A} 00+\mathrm{BST}+\% 2805 \% 3 \mathrm{~A} 00-07 \% 3 \mathrm{~A} 00+\mathrm{EDT}$ $\% 29+$ for+essential+maintenance $>$. Accessed: Ago. 01, 2012.doi: 10.1111/j.1939-1676.2008.0126.x.

KANG, B. et al. Bilateral congenital deafness in a bull terrier; brainstem auditory evoked response findings. Journal of Veterinary Clinics, v.25, p.506-509, 2008.

KAY, R. et al. Hearing in the dog as assessed by auditory brainstem evoked potentials. Veterinary Record, v.114, p.81-84, 1984 Available from: <http://www.ncbi.nlm.nih.gov/pubmed/6719811>. Accessed: Ago. 01, 2012

LASMAR, A. et al. Topodiagnóstico das disacusias sensoriais: audiometria supraliminar. In: FILHO, O.L.; CAMPOS, C.A Tratado de otorrinolaringologia. São Paulo: Roca, 1994. p.560598.

LENT, R. Os sons do mundo: estrutura e função do sistema auditivo. In: Cem bilhões de neurônios: conceitos fundamentais de neurociência. São Paulo: Atheneu, 2002. p.242-270.

MARCONDES, M. Semiologia do sistema nervoso de pequenos animais. In: FEITOSA, F.L.F. Semiologia veterinária: a arte do diagnóstico. São Paulo: Roca, 2008. p.411-460.

MARSHALL, A.E. Brainstem auditory-evoked response of the nonanesthetized dog. American Journal of Veterinary Research, v.46, p.966-973, 1985. Available from: <http://www.ncbi.nlm.nih. gov/pubmed/4014849>. Accessed: Ago. 01, 2012.
MEIJ, B.P. et al. Relationship between latency of brainstem auditory-evoked potentials and head size in dogs. Veterinary Quartery, v.14, p.121-126, 1992. Available from: <http://www. ncbi.nlm.nih.gov/pubmed/1485399>. Accessed: Ago. 15, 2013.

MUNRO, K.J.; COX, C.L. Investigation of hearing impairment in Cavalier King Charles spaniels using auditory brainstem response audiometry. Journal of Small Animal Practice, v.39, p.2-5, 1997. Available from: $<$ http://www.ncbi.nlm.nih.gov/pubmed/9121129>. Accessed: Ago. 01, 2012.

MUNRO, K.J. et al. Normative auditory brainstem response data for bone conduction in the dog. Journal of Small Animal Practice, v.38, p.353-356, 1997. Available from: <http://www.ncbi.nlm.nih. gov/pubmed/9282342>. Accessed: Ago. 01, 2012.

MYERS, L.J. et al. Reference values of the brainstem evoked response of methoxyflurane anesthetized and unanesthetized dogs. Veterinary Research Communications, v.9, p.289-294, 1985. Available from: <http://www.ncbi.nlm.nih.gov/pubmed/4095921>. Accessed: Ago. 01, 2012.

POMA, R. et al. MRI measurements of the canine auditory pathways and relationship with brainstem auditory evoked responses. Veterinary and Comparative Orthopaedic Traumatology, v.3, p.238-242, 2008. Available from: <http://www.schattauer. de/en/magazine/subject-areas/journals-a-z/vcot/contents/archive/ issue/131/manuscript/9939.html>. Accessed: Ago. 01, 2012.

POMIANOWSKI, A.; ADAMIAK, Z. Bone-conducted brainstem auditory evoked response in a dog with total bilateral ear canal ablation: a case report. Veterinarni Medicina, v.55, p.39-41, 2010. Available from: <http://vri.cz/docs/vetmed/55-1-39.pdf $>$. Accessed: Ago. 01, 2012.

PONCELET, L.C. et al. Audiograms estimated from brainstem tone-evoked potentials in dogs from 10 days to 1.5 months of age. Journal of Veterinary Internal Medicine, v.16, p.674679, 2002. Available from: <http://www.ncbi.nlm.nih.gov/ pubmed/12465764>. Accessed: Ago. 01, 2012.

SAS Institute. SAS/STAT User's guide.Versão 9.2. Cary, NC, 2009.

STRAIN, G.M. Congenital deafness and its recognition. Veterinary Clinics of North America - Small Animal Practice, v.29, p.895-907, 1999. Available from: <http://www.lsu.edu/ deafness/VetClinNA.htm>. Accessed: Ago. 01, 2012.

STRAIN, G.M. Hereditary deafness in dogs and cats: causes, prevalence, and current research. In: TUFTS' CANINE AND FELINE BREEDING AND GENETICS CONFERENCE, 2003, Old Sturbridge, M.A. Available from: <http://www.lsu.edu/ deafness/Tufts.htm>. Accessed: Out.25, 2010.

STRAIN, G.M. Brainstem auditory evoked response (BAER). In: STRAIN, G.M. (Ed.). Deafness in dogs and cats. OxfordShire: Cabi, 2011.p.83-105.

WEBB, A.A. Brainstem auditory evoked response (BAER) testing in animals. Canadian Veterinary Journal, v.50, p.313-318, 2009. Available from: <http://www.ncbi.nlm.nih.gov/pmc/articles/ PMC2643461/>. Accessed: Ago.01, 2012.

WILSON, W.J.; MILLS, P.C. Brainstem auditory-evoked response in dogs. American Journal of Veterinary Research, v.66, p. 2177-2187, 2005

Ciência Rural, v.44, n.5, mai, 2014. 